\title{
Association of troponin level and age with mortality in 250000 patients: cohort study across five UK acute care centres
}

\author{
Amit Kaura, ${ }^{1}$ Vasileios Panoulas, ${ }^{1}$ Benjamin Glampson, ${ }^{1}$ Jim Davies, ${ }^{2}$ Abdulrahim Mulla, ${ }^{1}$ \\ Kerrie Woods, ${ }^{2}$ Joe Omigie, ${ }^{3}$ Anoop D Shah, ${ }^{4}$ Keith M Channon, ${ }^{2}$ Jonathan N Weber, ${ }^{1}$ \\ Mark R Thursz, ${ }^{1}$ Paul Elliott, ${ }^{1,5}$ Harry Hemingway, ${ }^{4,5}$ Bryan Williams, ${ }^{4}$ Folkert W Asselbergs, ${ }^{4}$ \\ Michael O'Sullivan, ${ }^{6}$ Rajesh Kharbanda, ${ }^{2}$ Graham M Lord, ${ }^{7}$ Narbeh Melikian, ${ }^{3}$ Riyaz S Patel, ${ }^{4}$ \\ Divaka Perera, ${ }^{8}$ Ajay M Shah, ${ }^{3}$ Darrel P Francis, ${ }^{1}$ Jamil Mayet ${ }^{1}$
}

For numbered affiliations see end of the article.

Correspondence to: J Mayet j.mayet@imperial.ac.uk (ORCID 0000-0002-4665-6422)

Additional material is published online only. To view please visit the journal online.

Cite this as: BMJ 2019;367:16055 http://dx.doi.org/10.1136/bmj.l6055

Accepted: 14 October 2019

\section{ABSTRACT}

OBJECTIVE

To determine the relation between age and troponin level and its prognostic implication.

DESIGN

Retrospective cohort study.

SETTING

Five cardiovascular centres in the UK National Institute for Health Research Health Informatics Collaborative (UK-NIHR HIC).

\section{PARTICIPANTS}

257948 consecutive patients undergoing troponin testing for any clinical reason between 2010 and 2017.

MAIN OUTCOME MEASURE

All cause mortality.

RESULTS

257948 patients had troponin measured during the study period. Analyses on troponin were performed using the peak troponin level, which was the highest troponin level measured during the patient's hospital stay. Troponin levels were standardised as a multiple of each laboratory's 99th centile of the upper limit of normal (ULN). During a median follow-up of 1198 days

\section{WHAT IS ALREADY KNOWN ON THIS TOPIC}

Troponin is the preferred biomarker for the diagnosis of acute myocardial infarction

Evidence shows a direct relation between the magnitude of troponin increase and mortality, albeit over a limited range of troponin levels

Data on the relation between troponin level and mortality across a full age spectrum are limited, particularly for the very elderly compared with young people

Clinicians generally assume that higher troponins signify higher mortality in all age groups

\section{WHAT THIS STUDY ADDS}

A raised troponin level was associated with a clinically important increased mortality, regardless of age, even if raised only slightly above normal, with the excess mortality associated with a raised troponin level concentrated in the first few weeks

An unexpected inverted $U$ shaped relation between troponin level and mortality was seen in patients admitted with an acute coronary syndrome

The paradoxical decline in mortality at very high troponin levels may be driven in part by the changing case mix as troponin levels increase; a higher proportion of patients with very high troponin levels received invasive management

(interquartile range 514-1866 days), 55850 (21.7\%) deaths occurred. A positive troponin result (that is, higher than the upper limit of normal) signified a 3.2 higher mortality hazard (95\% confidence interval 3.1 to 3.2) over three years. Mortality varied noticeably with age, with a hazard ratio of 10.6 (8.5 to 13.3) in 18-29 year olds and 1.5 (1.4 to 1.6) in those older than 90 . A positive troponin result was associated with an approximately 15 percentage points higher absolute three year mortality across all age groups. The excess mortality with a positive troponin result was heavily concentrated in the first few weeks. Results were analysed using multivariable adjusted restricted cubic spline Cox regression. A direct relation was seen between troponin level and mortality in patients without acute coronary syndrome (ACS, $n=120049$ ), whereas an inverted $U$ shaped relation was found in patients with ACS ( $n=14468)$, with a paradoxical decline in mortality at peak troponin levels $>70 x U L N$. In the group with ACS, the inverted $U$ shaped relation persisted after multivariable adjustment in those who were managed invasively; however, a direct positive relation was found between troponin level and mortality in patients managed noninvasively.

\section{CONCLUSIONS}

A positive troponin result was associated with a clinically important increased mortality, regardless of age, even if the level was only slightly above normal. The excess mortality with a raised troponin was heavily concentrated in the first few weeks.

STUDY REGISTRATION

ClinicalTrials.gov NCT03507309.

\section{Introduction}

An association between an increase in troponin level and plaque rupture, acute coronary obstruction, and reduced myocardial perfusion is well established. ${ }^{1-3}$ The troponin assay is recognised by American and European guidelines as the preferred biomarker for the diagnosis of acute myocardial infarction..$^{4-7}$

Over the past two decades, assays for troponin have undergone vast improvements, allowing fast detection of troponin at low levels with increased precision. ${ }^{89}$ These improved assays have led to a substantial increase in the number of patients with a positive troponin result of uncertain significance.

The troponin test has been used as a prognostic marker for all cause mortality in people suspected of having had a coronary event. ${ }^{10} 11$ Some evidence 
suggests a direct relation between the magnitude of the increase in troponin and mortality over a limited range of troponin levels. ${ }^{11}{ }^{12}$ Clinicians generally assume that higher troponin levels signify higher mortality. Additionally, it is often difficult to know how to manage elderly people when a high troponin level is detected. This dilemma is partly due to the uncertain prognostic implication of a positive troponin result in older people and partly to a disinclination to investigate those in this group aggressively.

Decisions about the management of people with small increases in troponin levels, particularly if they have a non-cardiac presentation, can be difficult. It would be helpful to understand the prognostic implications of these small increases compared with larger increases when choosing treatment.

We describe how the prognostic impact of an increased troponin level varies with age, particularly in very elderly people, and investigate the prognostic importance of very small troponin levels compared with larger levels.

\section{Methods}

The National Institute for Health Research Health Informatics Collaborative project was established to facilitate the re-use of routinely captured clinical data for translational research. We obtained data from five collaborating hospitals (Imperial College Healthcare, University College Hospital, Oxford University Hospital, King's College Hospital, and Guys and St Thomas' Hospital), all tertiary centres with emergency departments. The supplementary material shows the data acquisition and statistical analysis plan.

We enrolled patients who had troponin measured $(\mathrm{n}=257948)$ during the study period $(2010-17)$. For those with more than one hospital episode of care during which troponin was measured during the study period, only the first episode was eligible. A total of 134517 of the 257948 patients (52.1\%) admitted to hospital had ICD-10 (international statistical classification of diseases, 10th revision) discharge codes. We classified patients as having had an acute coronary syndrome (ACS) on the basis of the assigned ICD-10 codes (supplementary table S1). ${ }^{13}$ All other patients, who between them had 3383 different ICD-10 primary diagnostic codes, were classified as not having had ACS. We individually reviewed those classified as not having had ACS with the top 10\% of ICD-10 codes and classified them into diagnostic groups. This top $10 \%$ comprised $83.1 \%$ of all diagnoses in the group of patients without ACS.

Invasive management was defined as having angiography within three months of the peak troponin level. We used a retrospective cohort study design, and followed up patients until death or censoring on 1 April 2017. Life status was ascertained using routinely collected data on the NHS Spine application, which is linked to the Office for National Statistics and thereby to the national registry of deaths.

The study dataset comprised patients who had their troponin level measured at each of the five academic centres between 2010 (2008 for University College Hospital) and 2017. Analyses on troponin were performed using the peak troponin level, which was the highest troponin level measured during the patient's hospital stay. Supplementary figure S1 shows the distribution of the number of troponin measurements taken during the index hospital admission for each patient. For those with a single troponin measurement, we used the peak troponin value based on this measurement. For the other patients with more than one troponin test in the same hospital episode of care, we defined the peak troponin value as the highest of all the measurements. For those with multiple episodes of care during which troponin was tested, we used the first episode of care for analyses.

Each centre measured troponin I or troponin $\mathrm{T}$ using either contemporary or high sensitivity assays (supplementary table S2). These tests yielded results in different measurable ranges, with unique cut-off points for the 99th centile of the upper limit of normal (ULN).

In clinical practice, troponin levels are often dichotomised into positive (meaning above the ULN) or negative. Troponin levels may have a progressive relation with prognosis too. The shape of this relation is not known across the full spectrum of values, and thus making an assumption of a linear relation between mortality and troponin (or log troponin) might not be valid. For these reasons, we treated the data in four ways. Firstly, we standardised the results by using the ratio of the observed troponin value divided by the ULN for each troponin assay. Secondly, we dichotomised the results as either positive or negative based on the ULN for each troponin assay. Thirdly, we examined the relation between peak troponin level and mortality in a series of troponin level bands: $<0.50 .5-1,>1-2$, $>2$ 5, >5-10, >10-100, >100-1000, and >1000 $\times$ ULN. This allowed us to examine the association between mortality and troponin level without making an advance assumption. Participating hospitals gave numerical values to $0.5 \times$ ULN or lower. The statistical analysis plan was for the highest troponin band to be $>10000 \times$ ULN. Once the data were collected, however, it was noted that three of the assays had an upper cutoff point of $<10000 \times$ ULN. To avoid miscategorising patients with these results, we merged the $>1000$ $10000 \times$ ULN and $>10000 \times$ ULN bands to form the $>1000 \times$ ULN band so that patients would be in the correct troponin group. Fourthly, we used restricted cubic spline analyses to assess the relation between troponin level, on a continuous scale, and mortality.

\section{Statistical analysis}

Descriptive statistics are displayed as medians (interquartile ranges) for continuous variables and numbers (percentages) for categorical variables. We compared the age distribution of patients for whom troponin was measured with that of the general population using the World Health Organization population estimates for the UK in $2015 .{ }^{14}$ 


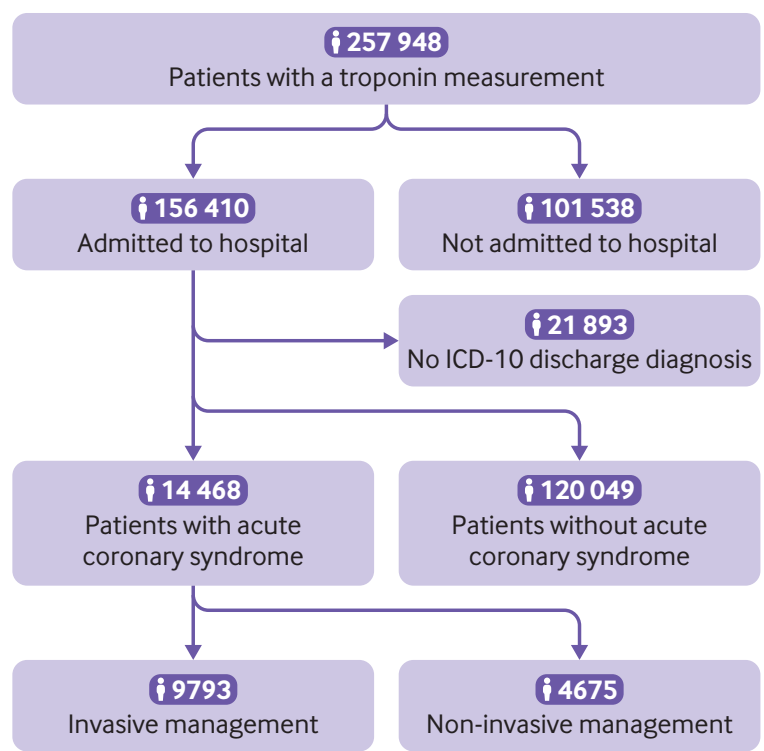

Fig 1 | Flow of study cohort. Patients were eligible for inclusion based on the first troponin measurement during the study period. ICD-10=international classification of diseases (10th revision) mortality, we again used Cox proportional hazards regression modelling. The effect of a positive troponin result on mortality was assessed at three years, and a landmark analysis was performed at three months. Thus analyses were restricted to patients who survived to three months. Cumulative mortality was displayed using corresponding Kaplan-Meier plots.

For non-linear relations, we used restricted cubic spline Cox regression analyses to model the association between all cause mortality and troponin level. Log transformation was used because of the positive skew of troponin values. Preliminary investigation suggested that four knots should be used to model troponin level in the restricted cubic spline analyses. We adjusted the splines for patient age; sex; haemoglobin level; creatinine level; $\mathrm{C}$ reactive protein level; white cell count; platelet count; the number of troponin tests during the index hospital admission; family history of cardiovascular disease; current smoker; diabetes mellitus; hypertension; hypercholesterolaemia; heart failure; previous ischaemic heart disease; atrial fibrillation; aortic stenosis; chronic kidney disease; neoplasm; and obstructive lung disease. For restricted cubic spline analyses, a hazard ratio of 1 was the reference value.

Means are presented with 95\% confidence intervals. Statistical analyses were performed using SPSS software version 24.0 (SPSS, Chicago, IL) or R 3.5.0 statistical package (R Core Team, Vienna, Austria). relation. To assess the association of dichotomous troponin level (above ULN or not) with all cause

Cox proportional hazards regression modelling was used to analyse the association between progressively higher troponin levels and all cause mortality. Thus no assumptions were made about the shape of the

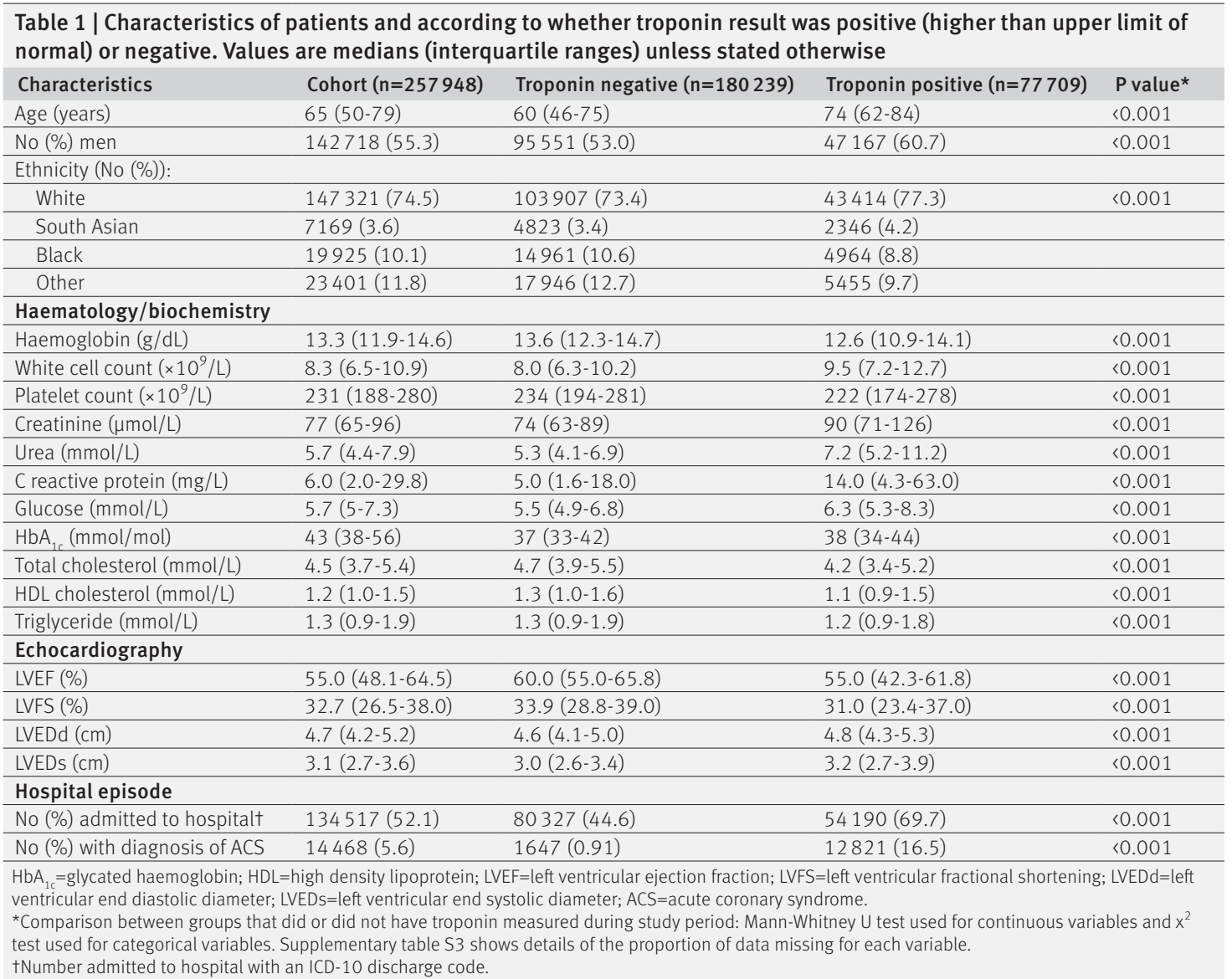


Patient and public involvement

This study involved the secondary use of existing data sources and did not include patients as study participants. No patients were involved in setting the research question, the study design, or the overall conduct of this study. Findings will be disseminated to a patient focus group to support discussions on how to develop the cardiovascular NIHR Health Informatics Collaborative theme. Supplementary appendix 2 provides a lay summary of the study.

\section{Results}

A total of 257948 patients had troponin levels measured during the study period (2010-17). Of 156410 (60.6\%) patients admitted to hospital with ICD-10 diagnostic codes, 14468 had a recorded diagnosis of acute coronary syndrome (ACS). Figure 1 shows the flow of the study cohort.

For patients without a diagnosis of ACS, the top $10 \%$ of ICD-10 codes (ie, most frequently recorded) were reviewed and classified into diagnostic groups (supplementary table S4). Among these patients reviewed, the coded diagnosis was cardiovascular in $20.2 \%$, non-cardiovascular in $37.7 \%$, and a broad range of undiagnosed multisystem symptoms in the remaining patients. The most common cardiovascular diagnoses among all patients without ACS were chronic ischaemic heart disease $(4.1 \%)$, atrial fibrillation or flutter $(2.6 \%)$ and heart failure $(2.4 \%)$. Pneumonia or lower respiratory tract infection (6.5\%), gastrointestinal disorders (4.2\%), and malignancy
(3.7\%) were the most common non-cardiovascular diagnoses in this cohort without ACS.

The median age was 65 years (interquartile range 50-79 years) and 142718 (55.3\%) of patients were men (table 1). The age distribution of the 257948 patients in the cohort was compared with that of the general population using WHO population estimates for the UK in 2015 (fig 2). The relative rate of patients who had troponin measured increased approximately linearly with age, from age 20 to 60, and then increased exponentially by about $50 \%$ for each decade of age thereafter (fig 2).

The proportion of patients with a positive troponin result increased progressively with age (fig 2), from $9 \%$ in the $18-29$ year age group to $50 \%$ in those older than 90 . The median positive troponin value was 2280 times higher than the median negative troponin level, and this relation was largely invariant with age.

Over a median follow-up of 1198 days (interquartile range 514-1866 days), 55850 (21.7\%) deaths occurred, 31112 (12.1\%) in the first year. The KaplanMeier three year mortality rates in the entire cohort was $20 \%$. Figure 2 shows the mortality rates for each age group.

\section{Relation between troponin positivity, age, and mortality}

A positive troponin result was associated with an overall 3.2-fold higher mortality hazard (95\% confidence interval 3.1 to 3.2 ) than a negative
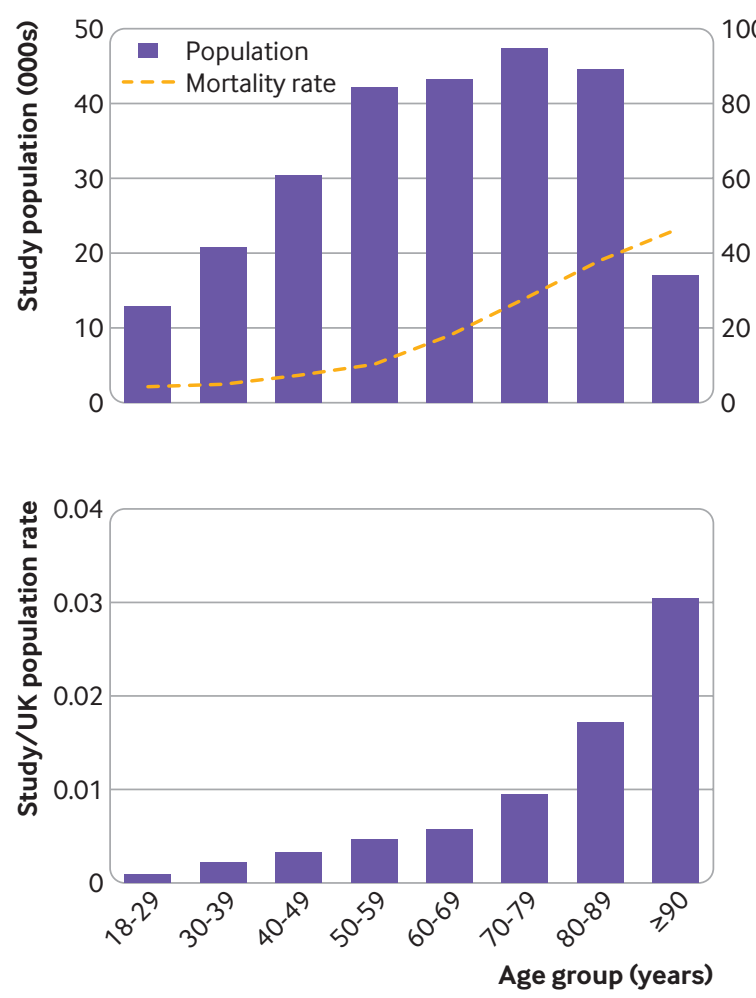
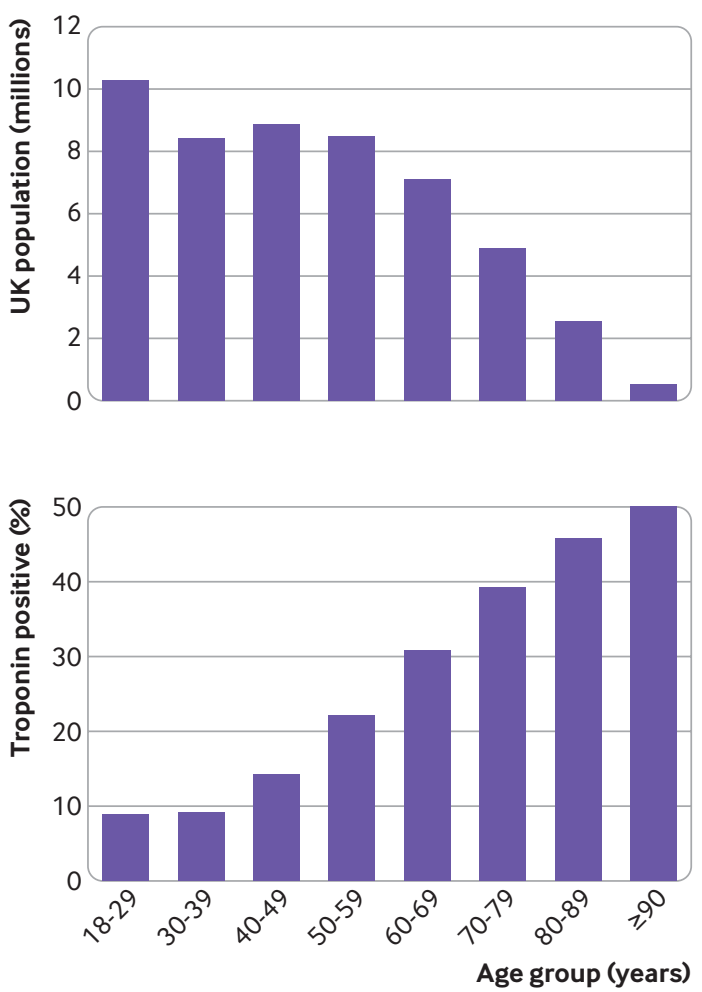

Fig 2 | Age distribution and three year estimated mortality rate in study population; age distribution of UK population; ratio of study to UK age distribution; and percentage of patients with raised troponin level across age groups 
troponin level over three years in the entire study cohort (Kaplan-Meier plot, supplementary fig S2; baseline characteristics for the troponin positive and troponin negative groups, table 1). This effect was particularly strong for young patients (18-29 years), with a mortality hazard ratio of 10.6 (95\% confidence interval 8.5 to 13.3 ). The effect declined progressively with age to a mortality hazard ratio of 1.5 (1.4 to 1.6) in those older than 90 (fig 3).

Figure 3 shows individual survival curves for troponin positive and negative groups for those aged $18-39,40-79$, and 80 or older. Supplementary figure S3 shows Kaplan-Meier survival curves for each age decade. In absolute terms, the increment in estimated three year mortality associated with troponin positivity ranged from 12.8 to 17.5 percentage points across all age groups, with a mean increment of 14.8 percentage points in all patients (fig 2). Most of the increase in mortality with a positive troponin result occurred within the first three months. Nevertheless, even when a landmark analysis was conducted at three months, a perceptible increase in mortality was found with a positive troponin result, which was present in all three age groups, and this grew steadily with time (supplementary fig S4).

\section{Distortion of the troponin-mortality link}

For all 257948 patients, we tested for the expected progressive relation between higher troponin levels and higher mortality (fig 4). Although mortality increased progressively up to about $>5-10 \times$ ULN, above this there was an unexpected progressive decline (fig 4).

Figure 4 shows the numbers of deaths in a series of time periods per 100 patients with an index troponin value in each of the troponin bands. The numbers are derived from the vertical increments in mortality from the Kaplan-Meier curves in figure 4. Those patients with a very high troponin level had high mortality up to one month later. Beyond that, mortality was similar to that of patients with troponin levels within the normal range.
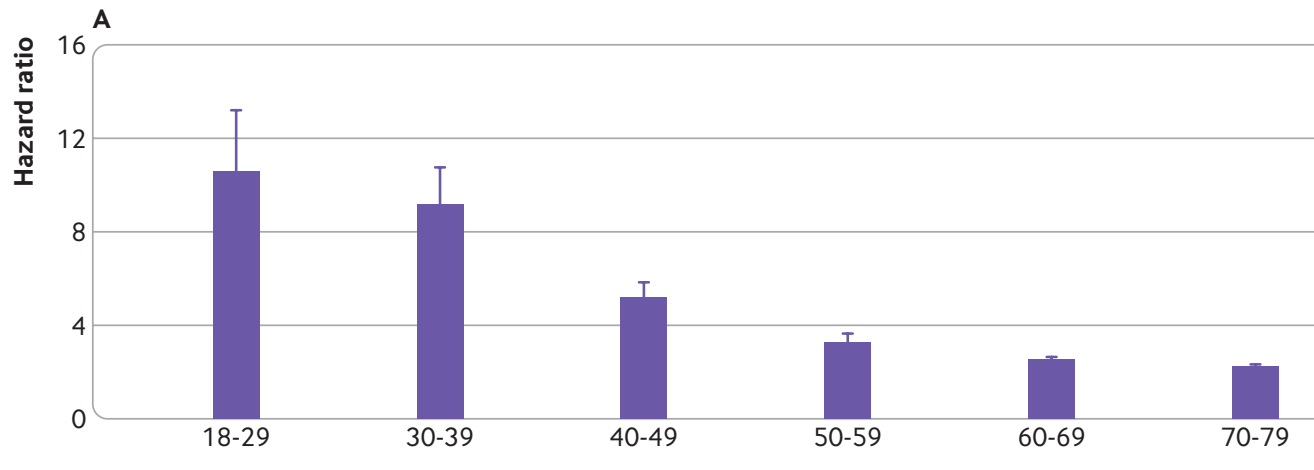

$80-89$

$\geq 90$
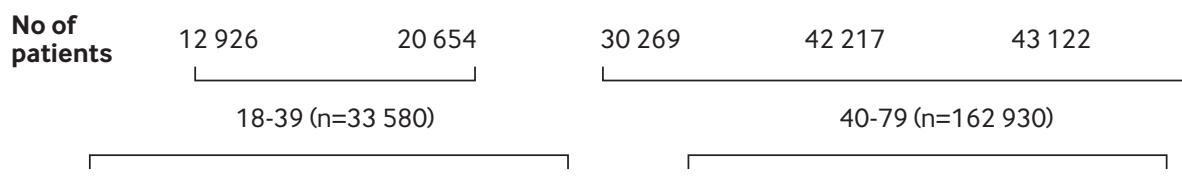

47322
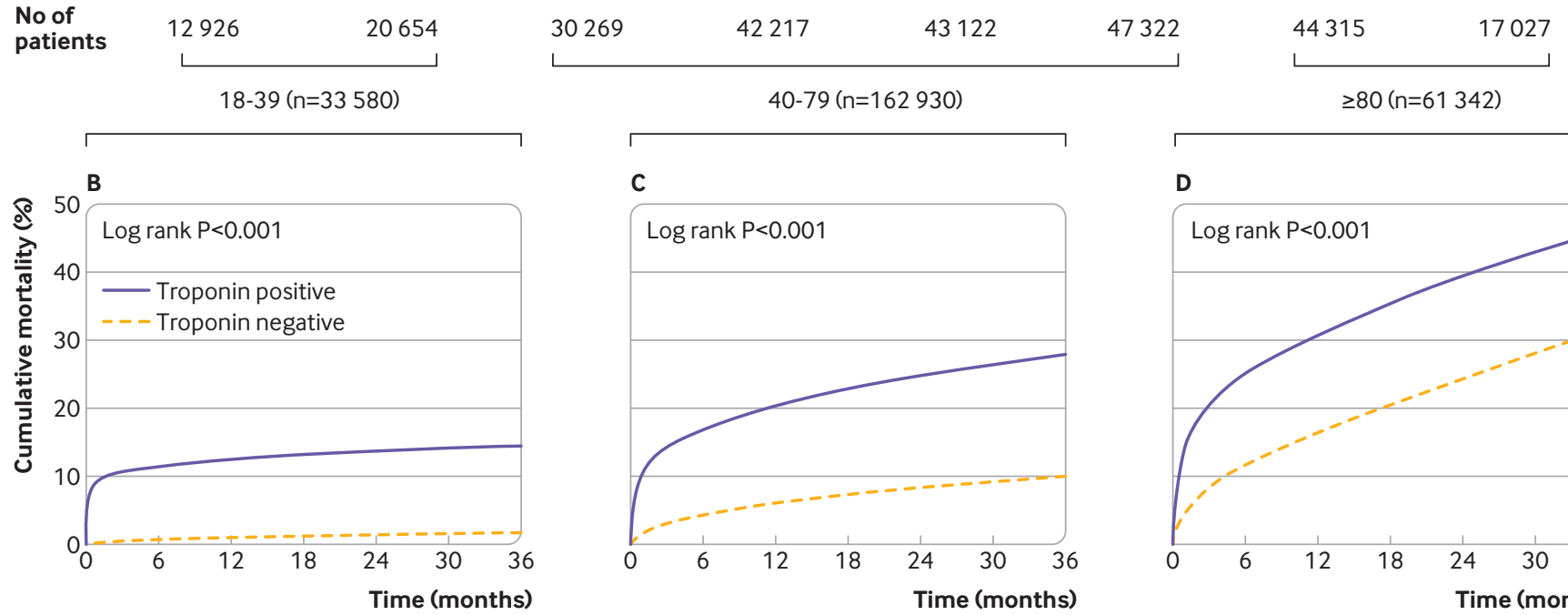

C

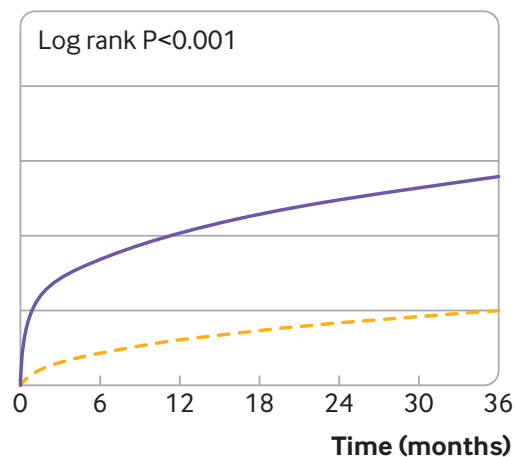

No at risk

Troponin positive

\begin{tabular}{|c|c|c|c|c|c|c|c|c|c|c|c|}
\hline 3132 & 2400 & 1996 & 1580 & 45763 & 33520 & 28115 & 22499 & 28792 & 18883 & 15122 & 11687 \\
\hline $304 \div$ & 26055 & 21118 & 16204 & 117167 & 101102 & 86054 & 70014 & 32550 & 115 & 4 & \\
\hline
\end{tabular}

Fig 3 | Hazard ratios for troponin positive versus troponin negative groups across different age bands for all patients and Kaplan-Meier mortality curves by troponin positivity in $18-39,40-79$, and $\geq 80$ years age bands. Error bars denote upper $95 \%$ confidence interval 
Supplementary table S2 provides details of the different troponin assays used by the five contributing centres. The inverted $U$ shaped relation was present across all troponin assays (supplementary fig S5) and was also seen when the first troponin level obtained during the index hospital admission was used.

Subgroup analyses were carried out on patients who were and were not admitted to hospital. The inverted $\mathrm{U}$ shaped relation was seen only in those who were admitted to hospital ( $\mathrm{n}=156410)$ (fig 5), with a peak hazard ratio of 2.4 (95\% confidence interval 2.3 to 2.4 ) at a peak troponin level of about $70 \times$ ULN. A direct positive relation was found between troponin level and hazard ratio across the full spectrum of troponin values for patients who were not admitted to hospital ( $\mathrm{n}=101538$ ) (supplementary fig S6). The Kaplan-Meier three year mortality rate was $14 \%$ in those not admitted to hospital, which was significantly lower than the rate of $23 \%$ seen in patients admitted to hospital. The numbers of patients not admitted to hospital in the $>1$ $10,>10-100,>100-1000$, and $>1000 \times$ ULN troponin bands were 9349 (9.2\%), 2209 (2.2\%), 1042 (1\%), and $380(0.4 \%)$, respectively.

\section{Relation between troponin level and mortality stratified by ACS diagnosis and invasive management}

The inverted $\mathrm{U}$ shaped relation between troponin band and mortality was found in patients both with $(n=14468)$ and without ACS $(n=120049)$ (supplementary fig S7). In a multivariable model, adjusting for key personal and clinical factors, the inverted $U$ shaped pattern between troponin level and mortality remained in patients with ACS (fig 6), but was a direct positive relation in those without ACS (fig 6).
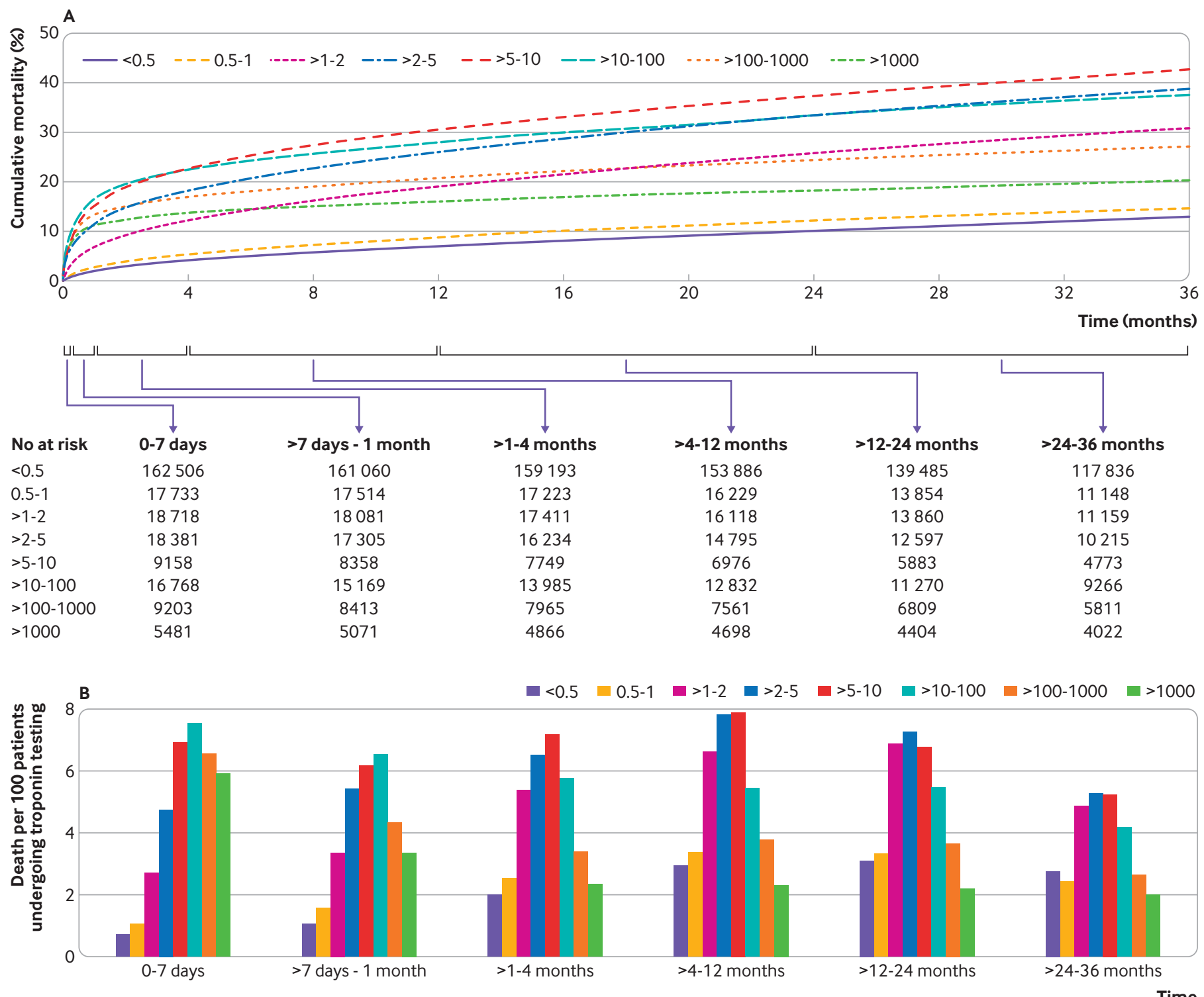

Fig 4 | Three year Kaplan-Meier cumulative mortality by troponin level for all patients, and deaths per 100 patients undergoing troponin testing during several time periods 
The rate of invasive management at three months was $67.7 \%(\mathrm{n}=9793)$ in the 14468 patients with ACS and $6.4 \%(n=7651)$ in the 120049 patients without ACS. The time to revascularisation in patients with ACS who underwent percutaneous coronary intervention or coronary artery bypass grafting was 1.3 days (95\% confidence interval 1.2 to 1.4 ) and 11.7 days (10.7 to 12.6), respectively. Among patients who did not undergo invasive management within three months of their first troponin measurement, 3.6\% of the group with ACS and 1.5\% of the group without ACS received it subsequently.

In patients with ACS, the rate of invasive management varied across the spectrum of troponin levels. The rate was only $39.4 \%$ for troponin levels $<1 \times$ ULN and $54.3 \%$ for those between 1 and $10 \times \mathrm{ULN}$. Above $10 \times \mathrm{ULN}$, the rate of invasive management increased to more than $90 \%$ for troponin levels $>1000 \times$ ULN (fig 7). After stratifying patients with ACS who were admitted to hospital according to whether or not they received invasive management, a restricted cubic spline Cox regression curve showed a progressive increase in mortality with higher troponin levels in the noninvasive management group, but this curve remained inverted in the invasive group (fig 7). Overall, invasive management was associated with lower hazard ratios than non-invasive management across all troponin levels in patients without ACS (supplementary fig S8).

\section{Discussion}

This study examines the implications of a raised troponin level in a large sample of patients undergoing troponin testing for any clinical reason across a wide range of ages. A raised troponin level is highly clinically meaningful, regardless of age, even if only slightly above normal. The inclusion of more than 250000 patients in this study allowed assessment of the prognostic power of troponin across a wide range of levels, and across the whole spectrum of ages. We found that even minor increases in troponin

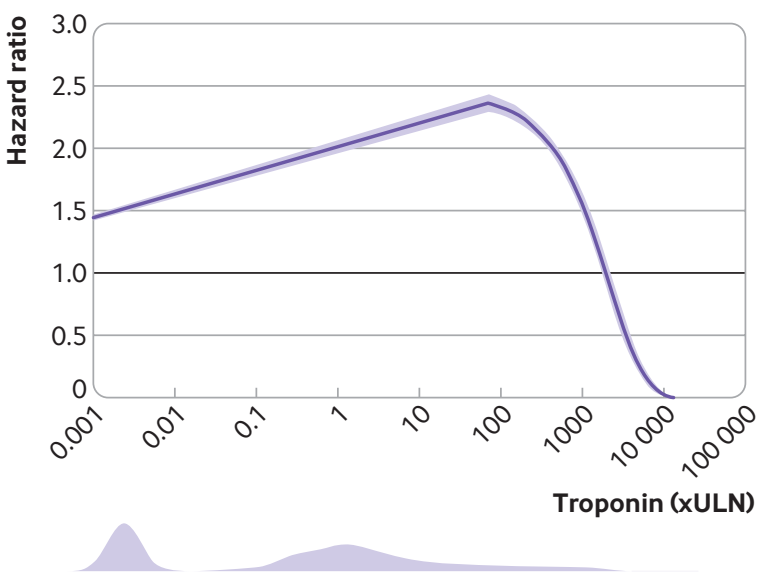

Fig 5 | Unadjusted association between peak troponin level and the hazard ratio for mortality for all patients admitted to hospital. The shaded area around the spline curve represents the $95 \%$ confidence interval. The probability distribution of troponin in all patients (pale purple) is displayed below the $x$ axis. The reference for the hazard ratio was set at 1 . ULN=upper limit of normal had major prognostic implications in patients both with and without acute coronary syndromes (ACS). An unexpected inverted $U$ shaped relation was found between troponin level and mortality in those patients admitted to hospital. For patients without ACS, multivariable adjustment led to reversion to the expected direct positive relation between troponin level and mortality. In the group with ACS, the inverted $\mathrm{U}$ shaped relation was present after multivariable adjustment in patients who were managed invasively, but reverted to a direct positive relation between troponin level and mortality in patients managed noninvasively.

Interpretation of troponin results in real life clinical practice, outside the confines of clinical trials, is often challenging. Troponin levels are measured in patients presenting to the emergency department, not only for chest pain but often for a wide variety of presentations. Consequently, sometimes the troponin test is positive in isolation, with no other indication to suggest myocardial ischaemia but with clear evidence of another diagnosis, such as pneumonia. ${ }^{15}{ }^{16}$ In such situations, clinicians may decide to focus on treating the primary diagnosis and not pursue the possibility of incidental or secondary coronary artery disease. Evidence suggests considerable under-recognition in hospital patients of cardiovascular disease and other conditions leading to fatal myocardial infarction. ${ }^{17}$

Additionally, relatively few finely stratified prognostic data are available in elderly patients. For such patients invasive treatment has not been clearly shown to lead to improved mortality compared with medical management. Because complication rates from procedures are higher in elderly patients, these patients are often managed conservatively.

\section{Relation between troponin positivity, age, and mortality}

We found that the hazard ratio for a positive troponin result attenuates markedly with age, from 10.6 in 18-29 year olds to only 1.5 in those older than 90 . The UK Myocardial Ischaemia National Audit Project (MINAP) registry found that a raised troponin level was associated with increased mortality in a selected cohort of patients with a diagnosis of ACS, including elderly patients. ${ }^{18}$

Our study including all patients who had their troponin measured, showed that a raised level is additive to age in predicting mortality even in very elderly patients ( $\geq 90$ years) and that young and middle age patients had much higher hazard ratios, particularly very young patients (18-29 years). Because of the high baseline risk in older patients, the absolute increment in three year mortality associated with a positive troponin result was remarkably consistent across all ages, at about 15 percentage points. This consistency is because the decline in hazard ratio with age is accompanied not by waning importance of troponin levels but by rising background mortality with age in people with troponin negative results. The absolute estimated three year mortality in people older 

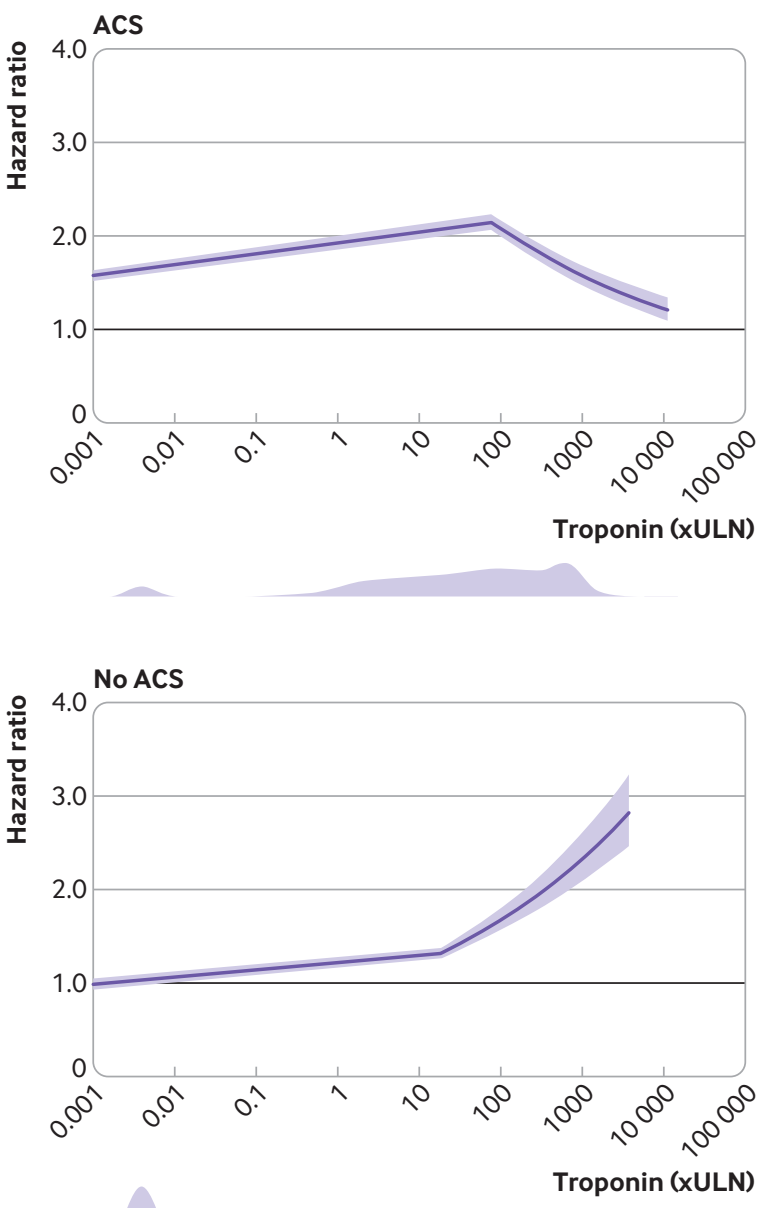

Fig 6 | Multivariable adjusted spline of association between peak troponin level and the hazard ratio for all cause mortality in subgroups with acute coronary syndrome (ACS) and those without ACS. Adjusted for patient age; sex; haemoglobin level; creatinine level; $C$ reactive protein level; white cell count; platelet count; number of troponin tests during the index hospital admission; family history of cardiovascular disease; current smoker; diabetes mellitus; hypertension; hypercholesterolaemia; heart failure; previous ischaemic heart disease; atrial fibrillation; aortic stenosis; chronic kidney disease; neoplasm; and obstructive lung disease. The shaded area around the spline curves represents the $95 \%$ confidence interval. Both curves arise from a single restricted cubic spline analysis using ACS diagnosis as a stratifier. The reference for the hazard ratio was set at 1 in both subgroups. The probability distribution of troponin level in patients with or without ACS is displayed below the $\mathrm{x}$ axes. ACS=acute coronary syndrome; ULN=upper limit of normal

than 80 with a positive troponin result was $46 \%$. Most of the mortality increment occurred within the first six months (fig 3). This pattern of early excess mortality in patients admitted to hospital with a raised troponin level was also seen in a US study of 25000 hospital patients. ${ }^{19}$

From this we can draw two conclusions. Firstly, regardless of age, a raised troponin level is an important clinical signal that should not be dismissed lightly. Secondly, as the excess mortality occurs early, a conservative wait and see approach may not be appropriate.

Distortion of the troponin-mortality link

The extent of the association between troponin level and mortality in patients with ACS has not been previously explored using troponin on a continuous scale. $^{3112021}$ Previous studies had sample sizes that were 35 -fold to 170-fold smaller. ${ }^{3112021}$ These small numbers led to studies grouping patients with troponin values above about $5 \times \mathrm{ULN}$, or more than $2.5 \%$ of the maximum detected troponin level. ${ }^{3} 112021$ The studies showed a relatively linear association between troponin level and mortality risk. Our study is large enough to allow analysis of high levels of troponin without grouping, across a population of patients who had troponin measured for clinical reasons.

Mortality increased from very low troponin levels to middle range levels, but above about $70 \times$ ULN a significant and progressive decline in mortality was seen in patients with a diagnosis of ACS. Patients with the very highest troponin levels had mortality comparable to those with normal troponin levels after the first month of follow-up. Possibly the paradoxical decline in mortality at very high troponin levels in patients with ACS may be due in part to a changing case mix as troponin levels increase. In addition, a higher proportion of patients with the highest troponin levels undergoes invasive management. In keeping with this, when we stratified patients with ACS according to invasive or non-invasive management, the inverted $U$ shaped relation between troponin level and mortality remained inverted in the group receiving invasive management; however, it became a direct positive relation in the non-invasive group.

Overall, invasive management was associated with lower hazard ratios than non-invasive management in patients with ACS and raised troponin levels. One explanation for this is that patients with underlying disease that lends itself to revascularisation and patients who are fit enough to undergo invasive management have a lower mortality than other groups. Possibly, revascularisation has improved prognosis in this patient group. Angiography, followed by coronary revascularisation with percutaneous coronary intervention or coronary artery bypass grafting, when appropriate, improves clinical outcomes, including cardiovascular mortality in patients with ACS. ${ }^{22}$ Findings from our study may reflect a substantial improvement in prognosis conferred by revascularisation in appropriate patients.

\section{Patients without ACS}

This study presents troponin data on a large cohort of patients without a diagnosis of ACS. Previous smaller studies were unable to categorise the upper troponin levels more finely and reported a progressive association between troponin level and mortality in the lower troponin bands. ${ }^{11} 1223$ A troponin rise in patients without ACS may be a marker of diminished organ level reserve as a result of global comorbidity rather than a specific pointer to unstable coronary disease. Consistent with this interpretation, one study reported acute myocardial infarction from obstructive coronary artery disease to be the cause of death in only $3 \%$ of such patients. ${ }^{11}$ 


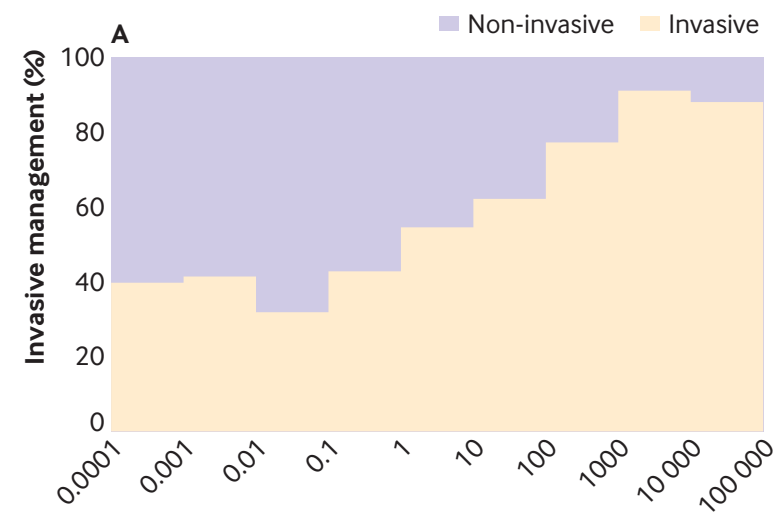

Troponin (xULN)

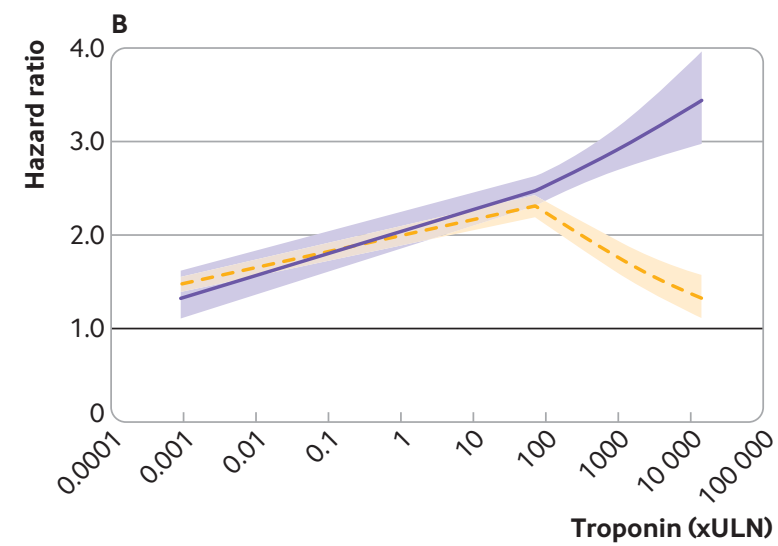

Fig 7 | Proportion of patients with acute coronary syndrome (ACS) undergoing invasive management according to troponin level; multivariable adjusted spline of association between peak troponin level and the hazard ratio for all cause mortality in patients with ACS stratified by invasive versus non-invasive management. Adjusted for patient age; sex; haemoglobin level; creatinine level; $C$ reactive protein level; white cell count; platelet count; number of troponin tests during the index hospital admission; family history of cardiovascular disease; current smoker; diabetes mellitus; hypertension; hypercholesterolaemia; heart failure; previous ischaemic heart disease; atrial fibrillation; aortic stenosis; chronic kidney disease; neoplasm; and obstructive lung disease. The grey shaded area around the spline curves represents the $95 \%$ confidence interval. Both curves arise from a single restricted cubic spline analysis using invasive management as a stratifier. The reference for the hazard ratio was set at 1 in the group with ACS who did or did not undergo invasive management. The probability distribution of troponin level in patients invasively managed (orange) and non-invasively managed (purple) is displayed below the $x$ axis of the bottom panel. ACS=acute coronary syndrome; ULN=upper limit of normal bands in patients without ACS. Despite this finding, there is currently no consensus on the best approach, whether non-invasively with imaging or invasively with coronary angiography, for establishing if patients without ACS but with raised troponin levels have underlying coronary artery disease. ${ }^{24}$

\section{Limitations}

This study benefits from having been conducted using real world clinical data from a large number of patients from multiple centres, but it has some limitations. The study was retrospective, with data extracted from electronic medical records and subject to the limitations of such an approach. Difficulties comprise the inability to account for all potential confounding factors, including the clinical factors prompting troponin measurement, such as patient symptoms or electrocardiogram findings, and drug treatment. Although our follow-up of survival was through the UK government's registry of deaths, this covers only people who continued to reside in the UK and not around $0.2 \%$ of people who emigrate each year.

The timing and frequency of troponin testing was clinically determined rather than standardised according to a trial protocol. Troponin is commonly measured in patients suspected of having ACS, but data on cardiac symptoms, such as chest pain or shortness of breath, were unavailable. Although we have no information on the reason for troponin measurement for individual patients, the study represents an important clinical subset because quite often we make decisions based on results of tests initiated by others. This study helps to inform a clinician receiving a troponin measurement result.

The five participating centres were all major acute centres with large emergency departments and cardiac units. Most patients have their troponin levels measured in an emergency department or acute medical ward, rather than a specialised cardiac unit. Despite this, there is still a potential for referral bias between those patients reviewed at the major acute care centres in our study and those seen in smaller district hospitals.

We had no information on whether receipt of evidence based cardiac care varied in different age groups. Finally, our study reported all cause mortality because this is incontrovertible and uniformly available. This study could not specify whether the cause of death was related to cardiac disease.

For patients without a diagnosis of ACS but with a raised troponin level, we were unable to distinguish acute from chronic myocardial injury accurately. This distinction is important, as the underlying disease mechanisms in acute and chronic myocardial injury are likely to differ. ${ }^{24}$ Both types of myocardial injury may occur in a variety of cardiac and non-cardiac illness (supplementary table S4). Our data and those of others suggest that many, but not all, patients without ACS but with an increased troponin level may not have unstable coronary artery disease. ${ }^{12}{ }^{25}$ One disease might have precipitated the other, or they could be coincidental. Invasive management was associated with lower cumulative mortality across all troponin

\section{Conclusions}

This study (TROP-RISK) used data from the contributing biomedical research centres in the NIHR Health Informatics Collaborative and presents data from more than a quarter of a million patients in routine clinical practice who undergo troponin measurement for clinical reasons. In young patients, a positive troponin result is associated with a very high mortality risk. Elderly patients have a very high mortality when the troponin level is negative, and even higher when the level is positive. A positive troponin result is therefore 
highly clinically meaningful, regardless of age, with the excess mortality associated with a raised troponin level being heavily concentrated in the first few weeks.

The assembly of sufficiently large sets of real world clinical data can cast light on patterns of disease that are impossible to define adequately in a single centre study or multicentre trial. Our analysis uncovered an unexpected inverted $U$ shaped relation between troponin level and mortality in patients with ACS. This relation may be driven in part by the changing case mix as the troponin level increases; a higher proportion of these patients undergo invasive management.

In a population of all patients in whom troponin is assessed for clinical reasons, this biomarker can risk stratify between high and low mortality risk for all age groups. Although even weakly raised troponin levels had marked prognostic significance, clinical decisions should depend on the underlying disease and not simply on the degree of increase in troponin.

\section{AUTHOR AFFILIATIONS \\ ${ }^{1}$ NIHR Imperial Biomedical Research Centre, Imperial College London and Imperial College Healthcare NHS Trust, Hammersmith Hospital, London W12 OHS, UK \\ ${ }^{2}$ NIHR Oxford Biomedical Research Centre, University of Oxford and Oxford University Hospitals NHS Foundation Trust, Oxford, UK ${ }^{3}$ NIHR Guy's and St Thomas' Biomedical Research Centre, King's College London and King's College Hospital NHS Foundation Trust,} London, UK

${ }^{4}$ NIHR University College London Biomedical Research Centre, University College London and University College London Hospitals NHS Foundation Trust, London, UK

${ }^{5}$ Health Data Research UK, London, UK

${ }^{6}$ NIHR Cambridge Biomedical Research Centre, University of Cambridge and Cambridge University Hospitals NHS Foundation Trust, Cambridge, UK

${ }^{7}$ Faculty of Biology Medicine and Health, University of Manchester, Manchester, UK

${ }^{8}$ NIHR Guy's and St Thomas' Biomedical Research Centre, King's College London and Guy's and St Thomas' NHS Foundation Trust, London, UK

This research has been conducted using National Institute for Health Research Health Informatics Collaborative (NIHR HIC) data resources. The NIHR HIC is a joint initiative between the NIHR Biomedical Research Centres at Imperial, Oxford, University College London Hospitals, Guy's and St Thomas', and Cambridge, which has provided data services, infrastructure, and expertise. The manuscript follows the STROBE guidelines for the reporting of observational studies.

Contributors: AK and VP contributed equally to this manuscript. AK, VP, DPF, and JM conceived the hypotheses and wrote the study protocol. BG, JD, AM, JO, and AK carried out the programming to extract the data from electronic healthcare records. AK, VP, and DPF undertook all data analyses. AK and VP drafted the manuscript. JM, DPF, AMS, DP, RSP, NM, GML, RK, MOS, FA, BW, HH, PE, MRT, JNW, $\mathrm{KMC}, \mathrm{ADS}, \mathrm{JO}, \mathrm{KW}, \mathrm{AM}, \mathrm{JD}$ and $\mathrm{BG}$ provided a critical review of the manuscript. All authors read and approved the final version of the manuscript. JM is guarantor for this paper. JM, RK, RSP, DP, and AMS were the leads for their respective institutions. The corresponding author attests that all listed authors meet authorship criteria and that no others meeting the criteria have been omitted, had full access to all the data in the study, and had final responsibility for the decision to submit for publication.

Funding: This paper reports independent research led and funded by the National Institute for Health Research (NIHR) Imperial Biomedical Research Centre (BRC), as part of the NIHR Health Informatics Collaborative with the NIHR Oxford BRC, the NIHR University College London Hospitals BRC, the NIHR Guy's and St Thomas' BRC, and the NIHR Cambridge BRC. RSP is funded by a British Heart Foundation Intermediate Fellowship. The views expressed in this publication are those of the authors and not necessarily those of the NHS, the National Institute for Health Research, or the Department of Health. PE and $\mathrm{HH}$ received Health Data Research (HDR) funding.
Competing interests: All authors have completed the ICMJE uniform disclosure form at www.icmje.org/coi disclosure.pdf and declare: support from the NIHR BRC for the submitted work; no financial relationships with any organisations that might have an interest in the submitted work in the previous three years; no other relationships or activities that could appear to have influenced the submitted work.

Ethical approval: This study was approved by the London-South East Research Ethics Committee (REC reference: 16/HRA/3327).

Data sharing: The datasets generated or analysed, or both during this study are not publicly available owing to ethical restrictions.

The guarantor (JM) affirms that this manuscript is an honest, accurate, and transparent account of the study being reported; that no important aspects of the study have been omitted; and that any discrepancies from the study as planned have been explained.

This is an Open Access article distributed in accordance with the Creative Commons Attribution Non Commercial (CC BY-NC 4.0) license, which permits others to distribute, remix, adapt, build upon this work non-commercially, and license their derivative works on different terms, provided the original work is properly cited and the use is non-commercial. See: http://creativecommons.org/licenses/ by-nc/4.0/.

1 Katus HA, Remppis A, Neumann F), et al. Diagnostic efficiency of troponin T measurements in acute myocardial infarction. Circulation 1991:83:902-12. doi:10.1161/01.CIR.83.3.902

2 Wong GC, Morrow DA, Murphy S, et al. Elevations in troponin T and I are associated with abnormal tissue level perfusion: a TACTICS-TIMI 18 substudy. Treat Angina with Aggrastat and Determine Cost of Therapy with an Invasive or Conservative Strategy-Thrombolysis in Myocardial Infarction. Circulation 2002;106:202-7. doi:10.1161/01. CIR.0000021921.14653.28

3 Lindahl B, Diderholm E, Lagerqvist B, Venge P, Wallentin L, FRISC II (Fast Revascularization during InStability in CAD) Investigators. Mechanisms behind the prognostic value of troponin $\mathrm{T}$ in unstable coronary artery disease: a FRISC II substudy. I Am Coll Cardiol 2001;38:979-86. doi:10.1016/S0735-1097(01)01501-7

4 Ibanez B, James S, Agewall S, et al, ESC Scientific Document Group. 2017 ESC guidelines for the management of acute myocardial infarction in patients presenting with ST-segment elevation: the task force for the management of acute myocardial infarction in patients presenting with ST-segment elevation of the European Society of Cardiology (ESC). Eur Heart J 2018;39:119-77. doi:10.1093/ eurheartj/ehx393

5 Roffi M, Patrono C, Collet JP, et al, ESC Scientific Document Group. 2015 ESC guidelines for the management of acute coronary syndromes in patients presenting without persistent ST-segment elevation: task force for the management of acute coronary syndromes in patients presenting without persistent ST-segment elevation of the European Society of Cardiology (ESC). Eur Heart / 2016;37:267-315. doi:10.1093/eurheartj/ehv320

6 Amsterdam EA, Wenger NK, Brindis RG, et al. 2014 AHA/ACC guideline for the management of patients with non-ST-elevation acute coronary syndromes: a report of the American College of Cardiology/American Heart Association Task Force on Practice Guidelines [correction in: I Am Coll Cardiol 2014:64:27134]. J Am Coll Cardiol 2014;64:e139-228. doi:10.1016/j. jacc.2014.09.017

7 O'Gara PT, Kushner FG, Ascheim DD, et al. 2013 ACCF/AHA guideline for the management of ST-elevation myocardial infarction: a report of the American College of Cardiology Foundation/American Heart Association Task Force on Practice Guidelines. J Am Coll Cardiol 2013:61:e78-140 doi:10.1016/j.jacc 2012.11.019

8 de Lemos JA. Increasingly sensitive assays for cardiac troponins: a review. JAMA 2013;309:2262-9. doi:10.1001/jama.2013.5809

9 Shah AS, Newby DE, Mills NL. High sensitivity cardiac troponin in patients with chest pain. BMJ 2013;347:f4222. doi:10.1136/bmj. patients

10 Chapman AR, Lee KK, McAllister DA, et al. Association of highsensitivity cardiac troponin I concentration with cardiac outcomes in patients with suspected acute coronary syndrome [correction in: JAMA 2018;319:1168.]. JAMA 2017;318:1913-24. doi:10.1001/ jama.2017.17488

11 Campbell AR, Rodriguez AJ, Larson DM, et al. Resource utilization and outcome among patients with selective versus nonselective troponin testing. Am Heart J 2018;199:68-74. doi:10.1016/j. ahj.2018.01.010

12 Roos A, Bandstein N, Lundbäck M, Hammarsten O, Ljung R, Holzmann MJ. Stable high-sensitivity cardiac troponin T levels and outcomes in patients with chest pain. I Am Coll Cardiol 2017;70:2226-36. doi:10.1016/j.jacc.2017.08.064

13 World Health Organization (WHO). International Statistical Classification of Diseases and Related Health Problems. 10th revision. 2016. https://icd.who.int/browse10/2016/en. 
14 Office for National Statistics (ONS). Population estimates for UK, England and Wales, Scotland and Northern Ireland. 2018. https://www.ons.gov.uk/peoplepopulationandcommunity/ populationandmigration/populationestimates/datasets/ populationestimatesforukengland andwalesscotlandandnorthernireland

15 Agewall S, Giannitsis E, Jernberg T, Katus H. Troponin elevation in coronary vs. non-coronary disease. Eur Heart/ 2011;32:404-11. doi:10.1093/eurheartj/ehq456

16 Hamm CW, Giannitsis E, Katus HA. Cardiac troponin elevations in patients without acute coronary syndrome. Circulation 2002;106:2871-2. doi:10.1161/01.CIR.0000044342.50593.63

17 Asaria P, Elliott P, Douglass M, et al. Acute myocardial infarction hospital admissions and deaths in England: a national followback and follow-forward record-linkage study. Lancet Public Health 2017:2:e191-201. doi:10.1016/S2468-2667(17)30032-4

18 Myint PK, Kwok CS, Bachmann MO, Stirling S, Shepstone L, Zaman MJ. Prognostic value of troponins in acute coronary syndrome depends upon patient age. Heart 2014;100:1583-90. doi:10.1136/ heartjnl-2014-305533

19 Winchester DE, Kline K, Estel C, Mahtta D, Taasan S, Peacock FW. Associations between cardiac troponin, mortality and subsequent use of cardiovascular services: differences in sex and ethnicity. Open Heart 2018;5:e000713. doi:10.1136/openhrt-2017-000713

20 Antman EM, Tanasijevic MJ, Thompson B, et al. Cardiac-specific troponin I levels to predict the risk of mortality in patients with acute coronary syndromes. N Engl/ Med 1996;335:1342-9. doi:10.1056/ NEJM199610313351802

21 Goldstein SA, Newby LK, Cyr DD, et al. Relationship between peak troponin values and long-term ischemic events among medically managed patients with acute coronary syndromes. J Am Heart Assoc 2017;6:e005334. doi:10.1161/JAHA.116.005334

22 Fox KA, Clayton TC, Damman P, et al, FIR Collaboration. Long-term outcome of a routine versus selective invasive strategy in patients with non-ST-segment elevation acute coronary syndrome a metaanalysis of individual patient data. J Am Coll Cardiol 2010;55:243545. doi:10.1016/j.jacc.2010.03.007

23 de Lemos JA, Drazner MH, Omland T, et al. Association of troponin T detected with a highly sensitive assay and cardiac structure and mortality risk in the general population. JAMA 2010;304:2503-12. doi:10.1001/jama.2010.1768

24 Chapman AR, Adamson PD, Mills NL. Assessment and classification of patients with myocardial injury and infarction in clinical practice. Heart 2017;103:10-8. doi:10.1136/heartjnl-2016-309530

25 Everett BM, Brooks MM, Vlachos HE, Chaitman BR, Frye RL, Bhatt DL, BARI 2D Study Group. Troponin and cardiac events in stable ischemic heart disease and diabetes. N Engl J Med 2015;373:610-20. doi:10.1056/NEJMoa1415921

Web appendix 1: Supplementary material Web appendix 2: Lay summary 